\title{
COMPLETUDE DAS CADERNETAS DE GESTANTES: REALIDADE DA REGIÃO NOROESTE DO PARANÁ
}

\section{COMPLETENESS OF THE BOOKLETS OF PREGNANT WOMEN: REALITY OF THE NORTHWEST REGION OF PARANÁ}

\section{COMPLETUD DE LAS CADERNETAS DE GESTANTES: REALIDAD DE LA REGIÓN NOROESTE DEL PARANÁ}

Emilli Karine Marcomini ${ }^{1}$, Ana Gabriela Fernande Frank ${ }^{2}$, Natália Namie Mizuguchi ${ }^{3}$, Adalberto Ramon Valderrama Gerbasi ${ }^{4}$, Nanci Verginia Kuster de Paul ${ }^{5}$

\section{RESUMO}

Objetivo: Este estudo tem por objetivo avaliar a completude do preenchimento das carteiras de gestante, verificando o preenchimento das informações por parte da equipe de saúde. Método: Trata-se de uma pesquisa de campo baseada em documentos de natureza quali-quantitativa, realizada em uma Maternidade da região noroeste do Paraná. Resultados: Realizou-se uma análise nos registros de perfil, antecedentes obstétricos, exame físico, consultas e exames. Os registros incompletos e a ausência de preenchimento das informações relevantes à saúde demonstra que existem falhas na assistência oferecida, bem como que o atendimento a gestante não está sendo integralizado e condizente com as recomendações vigentes. Conclusão: Evidencia-se que a carência de informações nas cadernetas de gestante interfere significativamente no desfecho final do processo gestacional e no resultado de toda assistência, sendo fundamental que os profissionais de saúde, estejam em conhecimento da real situação para que possam melhorar a qualidade de atendimento ofertada.

Descritores: Avaliação em saúde; Cuidado Pré-natal; Registros de enfermagem; Registros médicos.

\begin{abstract}
Objective: This study aims to evaluate the completeness of completing the pregnant woman's portfolios, verifying the completeness of the information by the health team. Method: This is a field research based on qualitative and quantitative documents, carried out in a Maternity from northwestern Paraná. Results: An analysis was performed in the profile records, obstetric history, physical examination, consultations and examinations. Incomplete records and the lack of completeness of information relevant to health demonstrates that there are failures in the care offered, as well as that care for pregnant women is not being paid and consistent with current recommendations. Conclusion: It is evident that the lack of information on pregnant woman's handbooks interferes significantly in the final outcome of the gestational process and in the outcome of all care, being essential that health professionals are aware of the real situation so that they can improve the quality of care service offered.

Descriptors: Health assessment; Prenatal care; Nursing records; Medical records.

1 Discente do Curso de Enfermagem da Universidade Paranaense (UNIPAR).

2 Discente do Curso de Enfermagem da Universidade Paranaense (UNIPAR).

3 Discente do Curso de Enfermagem da Universidade Paranaense (UNIPAR).

4 Graduado em Matemática (UEM); Mestre em Inovação Educativa (Universidade Autónoma de Barcelona, Espanha); Doutor em Inovação Educativa (Universidade Autónoma de Barcelona). Escritor e ganhador do Prêmio Jabuti (2018), na categoria Ciências.

5 Graduada em Enfermagem (Pontifícia Universidade Católica do Paraná); Mestre em Engenharia de Produção (Universidade Federal de Santa Catarina); Coordenadora geral de enfermagem da Associação Beneficente de Saúde do Noroeste do Paraná e professora adjunto da Universidade Paranaense.
\end{abstract}




\section{RESUMEN}

Objetivo: Este estudio tiene como objetivo evaluar la integridad de completar las carteras de la mujer embarazada, verificando la integridad de la información por parte del equipo de salud. Método: Esta es una investigación de campo basada en documentos cualitativos y cuantitativos, realizada en una Maternidad del noroeste de Paraná. Resultados: se realizó un análisis en los registros de perfil, antecedentes obstétricos, examen físico, consultas y exámenes. Los registros incompletos y la falta de integridad de la información relevante para la salud demuestra que hay fallas en la atención ofrecida, así como que la atención a las mujeres embarazadas no se paga y es consistente con las recomendaciones actuales. Conclusión: se evidencia que la falta de información en los manuales de las mujeres embarazadas interfiere significativamente en el resultado final del proceso gestacional y en el resultado de toda la atención servicio ofrecido.

Descriptores: Evaluación en salud; Cuidado prenatal; Registros de enfermería; Registros médicos.

\section{INTRODUÇÃO}

A gestação conceituada como um processo de transição corporal e emocional, é uma etapa complexa, transformadora e dinâmica, no qual a mulher vivencia momentos de prazer, dúvidas e ansiedade, devendo estar alicerçada em uma base de apoio que proporcione o encorajamento nas inseguranças que poderá vivenciar. ${ }^{1}$

Este alicerce é obtido durante a realização do pré-natal, por se tratar de uma fase de intenso acompanhamento de saúde, cuja assistência minimiza os anseios presentes durante o ciclo gravídicopuerperal. $^{2}$ No entanto, observa-se que a qualidade de assistência maternal-infantil encontra-se fragilizada, principalmente em decorrência das falhas nos registros de assistência. $^{3}$

Dessa maneira, é importante ressaltar que a caderneta da gestante é um meio de transcrição da assistência, realizada durante todo o ciclo gestacional, assim os registros são indispensáveis para manter um pré- natal de qualidade, devendo constar todas as condutas e os procedimentos realizados, para que os profissionais envolvidos tenham conhecimento do caso. ${ }^{4} \mathrm{O}$ cartão da gestante é um documento que permite aos profissionais identificar os fatores de risco, as condições de saúde da mulher, proporcionando o armazenamento das informações tanto durante a gestação como no processo de parto e posteriormente no puerpério, enriquecendo a assistência. ${ }^{5}$

A avaliação das cadernetas consiste em um processo de suma importância para analisar a qualidade pré-natal, uma vez que por meio delas pode-se identificar falhas na assistência bem como registros parciais de atendimento. $^{4}$ A etapa inicial de preenchimento da caderneta inicia na primeira consulta de pré-natal, onde é realizado um cadastro no SISPRENATAL (Sistema de Monitoramento e Avaliação do pré-natal, parto e puerpério), que no seguimento da gestação irão sendo inseridos mais informações da mãe e seu 
bebê, até a chegada do processo de parto, onde constará dados da concepção e das condições de nascimento do recémnascido. ${ }^{5}$ Pontua-se que os profissionais que estão envoltos da assistência gestacional, apesar de saber da importância dos registros, não valorizam a importância da caderneta preenchida corretamente, o que implica no processo de cuidados, dificultando a comunicação entre a equipe de saúde e interferindo na qualidade do atendimento prestado. ${ }^{4}$

Assim, é fundamental repensar em estratégias que reduzem os lapsos relativos ao pré-natal, bem como medidas que trazem o preenchimento da assistência como uma prática indispensável ao cuidado da gestante. ${ }^{3}$ Desta forma, o objetivo do presente estudo é avaliar a completude das cadernetas de gestantes, verificando o preenchimento das informações por parte da equipe de saúde.

\section{METODOLOGIA}

Trata-se de uma pesquisa de campo, de natureza quantitativa, em que se utilizou da análise documental de carteiras gestacionais. Este estudo foi realizado durante o período de 01 de abril de 2018 a 30 de junho de 2018, em uma Maternidade localizada na região noroeste do estado do Paraná.

Para a coleta de dados, empregou-se um formulário específico, elaborado especialmente para esta pesquisa e desenvolvido de acordo com a preconização estabelecida na Linha Guia da Rede Mãe Paranaense em sua sexta edição, as quais envolvem o perfil da gestante, a estratificação de risco, os antecedentes obstétricos, número de consultas e exames a serem realizados. ${ }^{6}$ Ressalta-se que foram avaliados todos os registros do processo gestacional na caderneta, envolvendo o prénatal, parto e puerpério.

Foram incluídas todas as puérperas que estavam aguardando a alta hospitalar, concordaram em participar do estudo assinando o TCLE (Termo de Consentimento Livre e Esclarecido) e permitiram o acesso das pesquisadoras em sua caderneta gestacional. A população alvo consistiu de todas as gestantes internadas no período da pesquisa e que atendiam aos critérios de inclusão. Assim, a amostra final foi de 244 cadernetas gestacionais.

Para a análise dos dados, as informações coletadas foram transcritas a uma planilha eletrônica, onde posteriormente foram analisados os dados quantitativos e realizou-se a elaboração de tabelas e gráficos.

Os dados foram coletados somente após a aprovação do Comitê de Ética em Pesquisa Envolvendo Seres Humanos da UNIPAR (Universidade Paranaense), sob parecer $n^{\circ}$. 2.214.786, conforme a resolução 
vigente $\mathrm{n}^{\mathrm{o}}$. 466/2012 disposta pelo Conselho Nacional de Saúde (CNS).

\section{RESULTADOS E DISCUSSÃO}

A caderneta da gestante é um instrumento de informação e comunicação da equipe, que favorece o cuidado na assistência pré-natal, por possuir dados significativamente importantes durante todo o processo gestacional. Deste modo, o preenchimento e o registro de cada atendimento efetuado contribuem para a elevação da qualidade da assistência, reduzindo a probabilidade de intercorrências e riscos severos à saúde materna-fetal. ${ }^{5}$

Em se tratando do perfil da mulher, a faixa etária em que se obteve o maior percentual foi a de 19 a 30 anos $(63,5 \%)$, conforme ilustra o gráfico a seguir. De acordo com a Estratificação de Risco estabelecido na Linha Guia da Rede Mãe Paranaense (sexta edição) ${ }^{6}$, apenas as gestantes com idade superior a 40 anos são qualificadas como risco intermediário, no entanto, obteve na então pesquisa poucas mulheres que possuíam esta faixa etária.

Ressalta-se que a gestação tardia é um agravante que pode comprometer a saúde do binômio mãe e feto, além disso, é uma tendência, cada vez mais presente no mundo, em decorrência da incorporação feminina do mercado de trabalho, das questões de estudos e melhoria de métodos de concepção. ${ }^{7}$

Gráfico 1 - Faixa etária das gestantes atendidas na maternidade da região noroeste do Paraná, 2018.

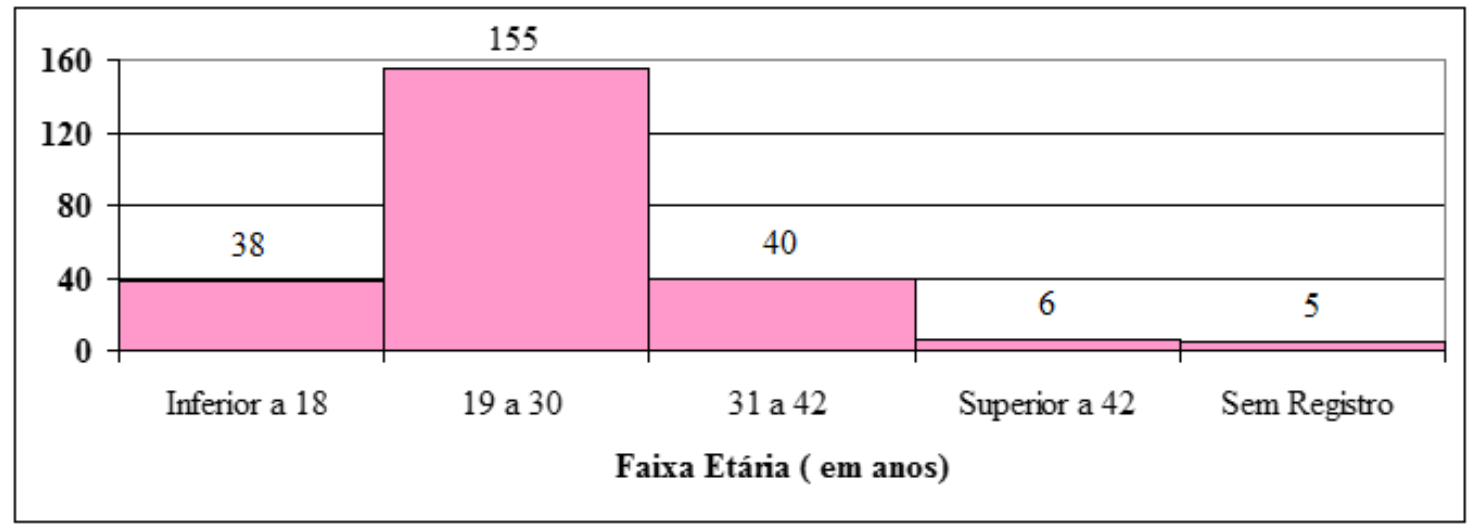

Fonte/ Ilustração: os autores, 2018.

No que tange a escolaridade, pode-se analisar na tabela abaixo que houve um destaque para o ensino médio completo, em que se obteve percentual de $34,83 \%$. Enfatiza-se que a escolaridade reflete diretamente no grau de entendimento da mulher em relação ao seu estado de saúde e aos cuidados na gestação, além de ser um indicador que estratifica a gestante em risco 
intermediário quando esta é analfabeta ou

possui menos de 3 anos de estudo. ${ }^{6}$

Tabela 1 - Nível de escolaridade das gestantes atendidas na maternidade da região noroeste do Paraná, 2018.

\begin{tabular}{l|c|c|c}
\hline \multicolumn{1}{|c|}{ Nível } & Completo & Incompleto & Total \\
\hline Não alfabetizada & 1 & - & 1 \\
Fundamental & 34 & 35 & 69 \\
Médio & 85 & 67 & 152 \\
Superior & 8 & 11 & 19 \\
Sem registro & 3 & - & 3 \\
\hline Total & $\mathbf{1 3 1}$ & $\mathbf{1 1 3}$ & $\mathbf{2 4 4}$ \\
\hline
\end{tabular}

Fonte/ Tabulação: os autores, 2018.

Quanto ao estado civil das gestantes, grande parte $(66 \%)$ das mulheres eram casadas ou possuem um companheiro fixo, $20 \%$ eram solteiras, $0,40 \%$ eram viúvas e $14 \%$ estavam com o registro em branco. Salienta-se que a participação paterna durante a gestação, proporciona uma maior aproximação do casal, elevando a segurança da mulher, e consequentemente contribuindo para o aumento dos vínculos durante a gravidez. ${ }^{8}$

No que corresponde a raça, identificou-se que a maior parte das mulheres pertenciam a cor parda $(39,34 \%)$, $33,60 \%$ das gestantes eram brancas, $10,24 \%$ eram negras, 0,40\% amarela, além de 16,66 em que não dispunham de registros relacionados a cor. Assim como em algumas características descritas anteriormente, a raça negra ou indígena é um indicador que estratifica a gestante como risco intermediário, devido ao maior risco da mortalidade infantil em negras, bem como doenças como a anemia falciforme. ${ }^{9}$

Outro dado de extrema relevância é o histórico gestacional, que influi diretamente na estratificação de risco da gestante e no decorrer do processo gestacional e do parto. Ao avaliar as carteiras, foi possível se deparar com uma grande negligência nas anotações referentes aos antecedentes obstétricos, conforme mostra a tabela 2 .

Tabela 2 - Antecedentes obstétricos das gestantes atendidas na maternidade da região noroeste do Paraná, $2018^{(*)}$.

\begin{tabular}{l|l|l|c|c}
\hline Antecedente & Sim & Não & Sem registro & Total \\
\hline Aborto & 36 & 83 & 125 & $\mathbf{2 4 4}$ \\
Parto normal & 49 & 71 & 124 & $\mathbf{2 4 4}$ \\
Cesárea & 72 & 55 & 117 & $\mathbf{2 4 4}$ \\
Gravidez ectópica & 0 & 49 & 195 & $\mathbf{2 4 4}$ \\
Imuno RH & 1 & 48 & 195 & $\mathbf{2 4 4}$ \\
\hline
\end{tabular}

Fonte/ Tabulação: os autores, 2018. 
A relevância do preenchimento dos antecedentes está no fato de ser uma informação que podem classificar a mulher em um grau de risco intermediário, como é o caso de um histórico anterior de aborto, natimorto ou óbito. Quantifica-se somente no antecedente de aborto que 51,22\% das cadernetas estavam com os registros em branco. Somado a isso, a questão das vias de parto, é mais um fator que não se obteve registros significativos (Parto normal 50,80\%; Cesariana - 46,72\%). Essa omissão de dados demonstra que há falhas na assistência oferecida ou ainda que o atendimento a gestante é voltado apenas para alguns aspectos, não utilizando do princípio da integralidade do cuidado.

A tabela acima apresenta ainda os registros de exames físicos, que devem ser feitos de modo céfalo-caudal e analisado criteriosamente pelo profissional de saúde. De acordo com o Manual do cuidado prénatal e puerpério, o exame físico é um agrupamento de técnicas (inspeção, palpação, percussão e ausculta) realizado pelos profissionais à gestante, em busca de identificar sinais relativos a doenças ou fatores que comprometam o bem-estar materno-fetal, assim, recomenda-se que seja realizado na primeira consulta um exame detalhado e nas subsequentes, um exame dirigido aos sistema que apresentarem queixa e registrar no cartão da gestante. ${ }^{10} \mathrm{~A}$ avaliação do enfermeiro no exame físico, proporciona a identificação de fatores de risco que podem interferir no decorrer da gestação, favorecendo a detecção de alterações sugestivas. ${ }^{11}$

Ao mensurar o percentual deste fator, $63,93 \%$ das gestantes não obtinham de um exame físico completo, o que implica gravemente no decorrer de toda assistência. Pode-se infligir que muitas vezes o profissional realizou o exame, entretanto não fez o registro na caderneta, implicando desta forma na qualidade de toda a assistência e enfatizando as lacunas existentes no cuidado gestacional.

No que concerne, ao número de consultas registradas no cartão da gestante, considerou-se apenas o mínimo de consultas (7), em conformidade com o preconizado pela Rede Mãe Paranaense.

Tabela 3 - Consultas realizadas pelas gestantes atendidas na maternidade da região noroeste do Paraná, 2018.

\begin{tabular}{c|l|l|l|l|l|l|l|l|l}
\hline Consultas & Data & Risco & Peso & IMC & PA & IG & $\begin{array}{l}\text { Altura } \\
\text { uterina }\end{array}$ & BCF & $\begin{array}{l}\text { Profissional } \\
\text { de saúde }\end{array}$ \\
\hline C1 & 234 & 199 & 215 & 149 & 227 & 185 & 152 & 149 & 107 \\
C2 & 232 & 183 & 216 & 91 & 230 & 203 & 171 & 189 & 87 \\
C3 & 220 & 185 & 206 & 1 & 212 & 209 & 201 & 196 & 76
\end{tabular}




\begin{tabular}{l|l|l|l|l|l|l|l|l|l} 
C4 & 234 & 183 & 199 & 73 & 212 & 198 & 202 & 206 & 73 \\
C5 & 210 & 164 & 195 & 64 & 208 & 205 & 201 & 206 & 14 \\
C6 & 196 & 167 & 193 & 69 & 206 & 200 & 193 & 201 & 66 \\
C7 & 169 & 156 & 175 & 63 & 181 & 170 & 183 & 180 & 60 \\
\hline
\end{tabular}

Fonte/ Tabulação: os autores, 2018.

É durante as consultas clínicas, que se estratifica a gestante conforme os achados presentes no momento, assim a cada encontro o patamar, ou seja, a classificação de risco a qual a gestante se encontra pode ser alterado. ${ }^{6}$ Em relação a estratificação de risco foi observado que é de maior realização nas primeiras consultas, entretanto é de fácil percepção a oscilação em todos os dados lançados na tabela 3, comprometendo a qualidade das consultas.

Juntamente com esse resultado é possível se deparar com disparidade encontrada na quantidade de consultas, onde houve média de quatro a cinco consultas por gestantes durante um período a termo normal de nove meses. Outros estudos evidenciam este fator, apontando uma baixa adesão em relação ao acompanhamento gestacional e número de consultas. $^{2}$

Notou-se que foram poucas as anotações identificadas com a categoria profissional na carteira, em 244 carteiras analisadas a média de profissional que anotou e identificou foi de 63,22\%, ressaltando que a maior parte foi registrada por enfermeiro(a). Correlacionando com outros estudos, uma parcela significativa de cadernetas não continha registros completos, inclusive atendimentos sem assinatura do profissional. ${ }^{4}$

Em se tratando dos exames laboratoriais recomendados na Linha Guia ${ }^{6}$, a gestante deve realizar o teste de gravidez ( $1^{\circ}$ trimestre), teste rápido para HIV ou Elisa $\left(1^{\circ}, 2^{\circ}, 3^{\circ}\right.$ trimestre), Teste rápido para sífilis $\left(1^{\circ}, 2^{\circ}, 3^{\circ}\right.$ trimestre $), \operatorname{VDRL}\left(1^{\circ}, 2^{\circ}, 3^{\circ}\right.$ trimestre), dosagem de proteinúria (somente quando necessário), tipagem sanguínea e fator $\mathrm{Rh}\left(1^{\mathrm{o}}\right.$ trimestre $)$, eletroforese de hemoglobina ( $1^{\mathrm{o}}$ trimestre), Urina I ( $1^{\circ}$ trimestres), hematócrito $\left(1^{\circ}, 2^{\circ}\right.$, $3^{\circ}$ trimestre), hemograma $\left(1^{\circ}, 2^{\circ}, 3^{\circ}\right.$ trimestre), HBS AG (1 ${ }^{\circ}$ trimestre), Ultrassonografia Obstétrica ( $1^{\mathrm{o}}$ trimestre), citopatologia cérvico-vaginal ( $1^{\circ}$ trimestre), toxoplasmose $\quad\left(1^{\mathrm{o}}, \quad 2^{\mathrm{o}}, \quad 3^{\mathrm{o}}\right.$ trimestre $)$, parasitologia de fezes ( $1^{\circ}$ trimestre).

Mediante esta listagem recomendada, é necessário que o profissional de saúde registre os valores dos resultados dos exames na carteira gestacional para que durante toda a assistência possa haver uma transmissão de informações de modo coerente. Entretanto, os resultados obtidos na pesquisa demonstram que o empenho em informar a realização/resultados dos 
exames é apenas verificado no primeiro trimestre (Gráfico 3). Correlacionando com os achados de outros autores ${ }^{3}$, que reforçam a maior ênfase na questão dos registros de exames apenas na primeira rotina, ficando os demais trimestres sem um preenchimento adequado.

Gráfico 2 - Exames feitos por trimestre pelas gestantes atendidas na maternidade da região noroeste do Paraná, 2018.

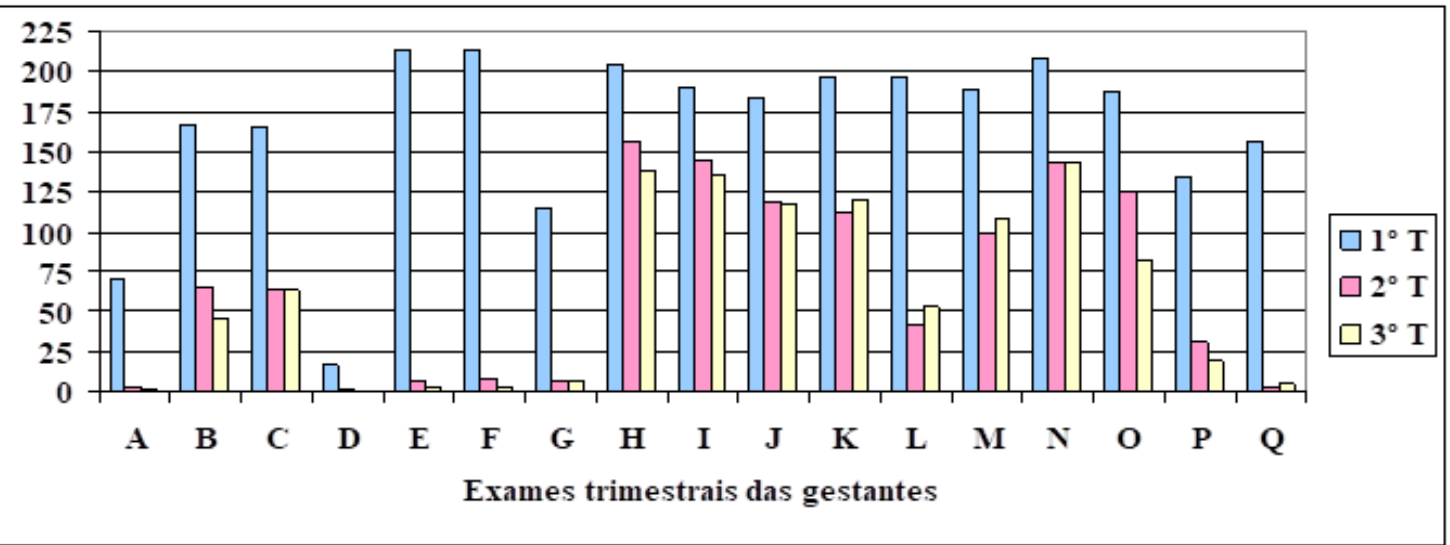

Legenda: Teste de Gravidez (A), Teste Rápido de HIV (B), Teste Rápido de Sífilis (C), Proteinúria (D), Tipagem Sanguínea (E), Fator RH (F), Eletroforese HB (G), Urina tipo I(H), VDRL (I), Hematócrito (J), Hemograma (K), Anti HBS (L), Elisa (M), Toxoplasmose (N), USG Obstétrica (O), Citopatologia (P), Parasitológico de Fezes (Q). Fonte/ Tabulação: os autores, 2018. 
Corroborando com os achados da presente pesquisa, outro estudo afirma que a realização dos exames gestacionais, não estavam em concordância com o preconizado pelas vigências do ministério da saúde ${ }^{2}$. Apenas alguns exames obtiveram uma média de 50\% de adequabilidade, como foi o caso da tipagem sanguínea/fator $\mathrm{Rh}, \mathrm{HBs}$ e Toxoplasmose.

Destacando a relevância do exame de tipagem sanguínea, o Manual do cuidado no pré-natal elucida que a incompatibilidade sanguínea ocasiona a formação de anticorpos maternos que iram agir contra o antígeno fetal, resultando em doenças que acarretaram danos à saúde tanto a mãe quanto ao feto, a exemplo da eritroblastose fetal. $^{10}$

Já em relação aos exames de hemograma e hematócrito, pesquisas apresentam uma frequência relativa de registros, tal como o de tipagem sanguínea, HIV e sífilis. No que tange ao HIV especificamente, a importância de se diagnosticar é que a transmissão pode ocorrer tanto na gestação, parto ou puerpério (aleitamento), evidenciando o maior risco de transmissão (65\%) durante o trabalho de parto e parto. ${ }^{3}$ Sobre a sífilis, o risco de transmissão se amplifica com o decorrer da gestação, à vista disso, orientase a realização do teste rápido para rastreamento, entretanto, devido as especificidades de cada região, disponibiliza-se o exame de VDRL. ${ }^{10}$

A respeito do exame parasitológico, quando não ocorre a sua realização no primeiro trimestre gestacional, compromete-se a saúde da mãe e o bemestar fetal, amplificando o risco de um recém-nascido prematuro e de baixo peso. ${ }^{10}$ Observa-se no gráfico 2 que embora $64 \%$ das gestantes tenha realizado o exame, uma parcela significativa (36\%) não apresenta registro no cartão pré-natal.

O acesso ao serviço de saúde é um obstáculo que interfere no diagnóstico precoce de comorbidades, no tratamento dos envolvidos, além de elevar a possibilidade de transmissão vertical ao feto. ${ }^{12}$ Portanto, coloca-se em ênfase a relevância de conhecer cada realidade e desenvolver estratégias para que a população esteja cada vez mais próxima do acesso a saúde.

Menciona-se ainda algumas dificuldades que interferem na realização do exame, como exemplo, o custeio dos exames por cada município, resistência dos médicos na solicitação e a forma de organização do processo laboratorial. ${ }^{13}$ Como equipe de saúde, deve-se implementar formas facilitar o acesso da 
população e minimizar os obstáculos que afastam a gestante do cuidado pré-natal.

Pontua-se que os exames tanto laboratoriais quanto de imagem, agregam fatores para o desenvolvimento de um prénatal adequado, assim, é necessário estabelecer estratégias que facilitem o acesso das gestantes tanto nas consultas quanto nos exames, por meio de transporte gratuito até o local de realização, recursos relativos a disponibilidade e agilidade, bem como elevação da cobertura dos exames no público em questão. ${ }^{13}$

Mediante todos os resultados encontrados que demonstram a carência de anotações em pontos específicos do cartão, ressalta-se que para um atendimento de qualidade e redução de indicadores de morbimortalidade infantil, é fundamental o aperfeiçoamento de ações que auxiliam na efetividade do pré-natal, melhorando a

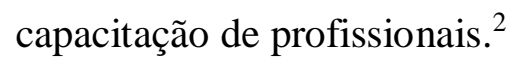

\section{CONCLUSÃO}

Ao observar uma carteira gestacional e verificar o quão carente de informações elas podem se apresentar, causa uma preocupação em como será o desfecho do ciclo gravídico/puerperal desta mulher, pois pode-se deduzir que o acompanhamento não foi realizado da forma correta.

Neste sentido, a pesquisa elucidou alguns aspectos relevantes do pré-natal que não estavam com preenchimento adequado, o que se relaciona diretamente com a qualidade de assistência oferecida.

Cabe ressaltar que os profissionais devem ser qualificados e comprometidos em cada novo encontro que tiverem com a gestante, anotando e realizando cada ação realizada, bem como cabe a futura mãe se preocupar e entender que sua adesão em todas as fases é primordial para a sua saúde e a de seu bebê.

Portanto, vale salientar a educação continuada bem como uma boa qualificação dos profissionais que entram em contato com o pré-natal, para realização de consultas gestacionais de qualidade e também para cativar a gestante a aderir ao seu acompanhamento periódico de forma prazerosa e consciente.

Sendo assim, conclui-se que a completude das cadernetas de gestante na região avaliada, apresenta-se falha, devendo os gestores estarem em conhecimento da real importância de cada registro para posteriormente identificação das mudanças necessárias.

1- Pio DAM, Capel MS. Os significados do cuidado na gestação. Rev Psicol Saúde 2015; 7(1):74-81. 
2- Balsells MMD, Oliveira TMF, Bernardo EBR, Aquino PS, Damasceno AKC,

Castro RCMB et al. Avaliação do processo na assistência pré-natal de gestantes com risco habitual. Acta Paul Enferm. 2018; 31 (3):247-54.

3- Queiroz DJM, Oliveira KCA, Soares DB. Avaliação da assistência pré-natal: relevância dos exames laboratoriais. Rev Bras Promoç Saúde. 2015; 28(4):504-12. 4- Oliveira CCSS. Pré-natal: análise dos registros quanto à adequação dos conteúdos na caderneta da gestante de risco habitual. [Monografia]. São Luiz, MA: Universidade Federal do Maranhão; 2018. 45 p.

5- Souza IA, Serinolli MI, Novaretti MCZ, Souza DCC. Compatibilidade entre os dados do cartão da gestante e o sistema informatizado da web sisprenatal.

Prisma.com [Internet]. 2016 [citado em 27 jan 2019]; 32:127-47. Disponível em: http://ojs.letras.up.pt/index.php/prismacom /article/view/2216

6- Secretaria de Estado da Saúde (Paraná). Linha guia: rede mãe paranaense. 6ed. [Internet]. Curitiba: SESA; 2017 [citado em 27 jan 2019]; 63p. Disponível em: http://www.saude.pr.gov.br/arquivos/File/ LinhaGuiaMaeParanaense_final_2017.pdf 7- Aldrighi JD, Wall ML, Souza SRRK Vivência de mulheres na gestação em idade tardia. Rev Gaúch Enferm. 2018; 39:1-9.

8- Ribeiro JP, Gomes GC, Silva BT, Cardoso LS, Silva PA, Strefling ISS. Participação do pai na gestação, parto e puerpério: refletindo as interfaces da assistência de enfermagem. Espaç Saúde [Internet]. 2015 [citado em 27 jan 2019]; 16(3):73-82. Disponível em:

http://espacoparasaude.fpp.edu.br/index.ph p/espacosaude/article/view/398/386 9-Ministério da Saúde (Brasil), Secretaria de Gestão Estratégica e Participativa, Departamento de Apoio à Gestão Participativa e ao Controle Social. Política nacional de saúde integral da população negra [Internet]. 3ed. Brasília, DF:

Ministério da Saúde; 2017 [citado em 27 jan 2019]. 44p. Disponível em:

http://bvsms.saude.gov.br/bvs/publicacoes/ politica_nacional_saude_populacao_negra _3d.pdf

10- Prefeitura Municipal de Londrina, Secretaria Municipal de Saúde. Manual do cuidado no pré-natal e puerpério na atenção primária em Saúde [Internet]. 2ed. Londrina, PR: Secretaria Municipal de Saúde; 2016 [citado em 24 jan 2019]. 434p. Disponível em:

http://www1.londrina.pr.gov.br/dados/ima ges/stories/Storage/sec_saude/protocolos_c linicos_saude/manual_cuidado_prenatal_puerperio_atencao_primaria_saude.p df

11- Lima LFC, Davim RMB, Silva RAR, Costa DARS, Mendonça AEO.

Importância do exame físico da gestante na consulta do enfermeiro. J Nurs UFPE on line [Internet]. 2014 [citado em 27 jan 2019]; 8(6):1502-9. Disponível em: https://periodicos.ufpe.br/revistas/revistaen fermagem/article/view/9838/10038 12- Lopes SGG. Exames no pré-natal: desenvolvimento de cartaz educativo para profissionais de saúde com ênfase na atenção à transmissão vertical de sífilis e HIV. [Monografia]. Florianópolis: Universidade Federal de Santa Catarina; 2014. 10 p.

13- Ferreira H, Novelli IA, França AFO, Caldeira S, Silva RMM. Rotina de exames na atenção ao pré-natal após a implantação do programa rede mãe paranaense. Rev Enferm UFSM. 2017; 7(4):685-99.

RECEBIDO: 14/02/2019

APROVADO: 24/09/2019

PUBLICADO: 12/2019 